\title{
Pain, Placebo, and Nocebo: Epistemic, Ethical, and Practical Issues
}

\author{
James Giordano, PhD, and Mark V. Boswell, MD, PhD
}

Of particular interest in this issue is the paper by Manchikanti, Pampati, and Damron (1), which examines putative placebo and nocebo effects observed while studying the influence of sedation on the diagnostic validity of facet nerve injections. The authors took a novel approach to investigating the role of the well-known placebo effect, as well as the less understood nocebo effect (where an agent, active or not, worsens the pain). In the first part of the study, they examined the effects of sedation on the validity of diagnostic facet nerve blocks (e.g., the rate of false positives). If strict outcome criteria were used, including $\geq 80 \%$ pain relief and the ability to perform previously painful movements without pain after intravenous "sedation," the diagnostic validity of controlled facet nerve blocks was preserved. The falsepositive rate, which included a placebo response, remained low $(<8 \%)$ and was generally clinically insignificant.

In the second part of the study, the authors examined the effects of sedation on subsequent patient experience with the procedure. At 3 months, patients were questioned about whether their experiences as compared to previous treatments were better, worse, or no different, and this was related to the type of intravenous injection given (i.e., normal saline, midazolam, or fentanyl). They were able to quantify the placebo response and the protocol was sufficiently sensitive to dis-

From: Center for Clinical Bioethics, Georgetown University Medical Center, Washington, DC; and Case School of Medicine and University Hospitals of Cleveland, Ohio.

Address Correspondence:

James Giordano, PhD

Center for Clinical Bioethics

Georgetown University Medical Center

4000 Reservoir Rd.

Washington, DC 20057

E-mail: Gsynapse22@aol.com tinguish placebo effect from possible analgesic effects of fentanyl, at least in the cervical facet group. In addition, the study demonstrated that up to $8 \%$ of patients had a worse experience compared to previous treatment, irrespective of the solution given intravenously, that is, a possible nocebo effect.

The reader may initially conclude that this was not actually a nocebo response given that a number of factors may have contributed to the patients' perceptions of a "worse" experience, including the 3 month interval since the procedure, the natural history of the underlying pain process, the technique used at the last treatment, as well as the type of sedation used. On the other hand, the average clinician would be comfortable with patients' descriptions of the amount of pain relief obtained with a previous procedure, even after an interval of 3 months. Indeed, this is the usual situation in clinical practice. That is, the decision to perform the next treatment often is based on the amount of pain relief derived from a previous treatment (and the satisfactory nature of the previous experience). In any case, the protocol used in the study approximates the real-world clinical situation in terms of assessing clinical outcomes. It is, therefore, reasonable to consider the observed responses as representing placebo and nocebo effects.

If one accepts the argument offered by the authors that the observed responses are indeed placebo and nocebo responses, what do the effects mean? The authors astutely note that these events represent patient-centered responses of differing "valence" (i.e., the positivity of placebo or negativity of nocebo), which can be distinguished from changes in underlying pathology and/or pharmacologic effects of the intravenous agents used. The randomized design in their study further supports these distinctions, and the pro- tocols used elucidate the potential for placebo and nocebo effects to occur in realworld clinical therapeutic circumstances.

It is this patient-centeredness that raises the importance of placebo and nocebo effects in a broader clinical context. In this study, all of the patients experienced function-limiting cervical or lumbar facet pain for a period of at least 1-2 years, and had positive responses to prior therapeutic facet joint nerve blocks. In addition, all of the patients were experiencing chronic pain with some disability, but it is difficult to assess how chronic pain affects the central nociceptive neuraxis of an individual patient. The plasticity of this system can be affected by numerous variables, including genomic factors (i.e., genotypic pre-disposition toward the expression of phenotypic characteristics that can affect pain sensation and perception) and environmental and psychosocial variables (e.g., prior exposure to pharmacologic agents, lifestyle, and sociocultural factors). Presumably, such effects can induce changes in the central nervous system from spinal cord to brain, and reciprocally affect brain regions subserving cognition, emotion, behavior and physiology (e.g., endocrine and immune function). Moreover, the extent to which particular brain regions are altered by pain differs for each person (2). While it may be fair to state that a particular neuroanatomical site is activated by pain, the effect of such activation on a particular person's sensations, perceptions, memories, and consciousness is individually variant and, therefore, unique. Thus, the ontological nature of a particular patient's pain assimilates variables from prior and current experience, and can influence expectation and meaning ascribed to pain and the clinical interaction(s) (3).

In many ways, this indicates a need to re-address how Cartesian thinking 
has been altered in modern medicine to maintain separation of objective physical processes from subjective, intangible, mental events in the conceptualization of disease, illness, and therapeutic intervention. Yet, interestingly, if we examine Descartes' work more thoroughly, we find that he posited that body and mind do indeed interact, and the site of this interaction is within the brain (although this was naively thought to occur at the pineal gland (4)). Three hundred fifty years later, this insight becomes increasingly notable in light of progress in experimental and philosophical neuroscience that has fostered a more monistic perspective. The body is no longer viewed as a system independent of mental processes, but is considered instead to be a unified entity such that body, brain, and mind exist as a complex, bi-directional system within a dynamic, networked hierarchy. Biological and psychosocial effects can combine to modify, and perhaps determine, the outcome of clinical treatment. Frequently, such effects are subtle and occur as a function of a particular person's experience of the clinical encounter. This concept is critical to an understanding of chronic pain, and placebo and nocebo effects.

It is this point that links the empirical basis of the work of Manchikanti, Pampati, and Damron to more epistemic, and ultimately to ethical issues. Contemporary medicine relies heavily on an evidence-based foundation for clinical decision-making and multiple domains of information contribute to the decision process. Pain is a subjective experience and we attempt to use technological means to identify underlying objective pathology that may explain the pain. Yet, an understanding of the subjective nature of pain is necessary in order to recognize that pain is a disease process and a subjective illness phenomenon in a particular patient $(5,6)$. Often, it is this realm of experience that most strongly contributes to patients' interpretations of pain, therapeutic expectations, and, indeed, placebo and nocebo effects. While the heuristic value of such information to the physician is obvious, concerns about the phenomenological aspects of a particular patient's pain may not "fit" into a protocol of purely technologic assessment. This requires a more thorough use of narrative. We posit that such narrative, together with an understanding of pain mecha- nisms and expression, can afford greater understanding of the multi-dimensional nature of a particular patient's pain, and thereby improve the decision process that will lead to the most appropriate treatment.

Herein lie several ethical issues: first, as a steward of knowledge, the physician is a therapeutic agent who must employ information to make technically "right" decisions, to render treatment based upon a mechanistic understanding of the pathologic process and therapeutic intervention at hand. However, what is technically "right" may not be biomedically or ethically "good" for a particular patient along the disease-illness continuum. Hence, the physician also acts in a moral capacity to render an ethically "good" treatment $(7,8)$. Certainly, one form of knowledge is scientific; contextually this involves identifying the neurological mechanisms and processes that subserve pain in all of its dimensions (i.e., sensory, perceptual, cognitive, etc.), and how these neural substrates can produce placebo and/or nocebo effects and responses. This knowledge is gleaned from research, and represents the factual basis of applied biomedicine. However, such a 'secular' understanding of neural function may be insufficient to fully appreciate the dilemma of pain for a particular patient and allow reasoning necessary to render a treatment that is biomedically and ethically good for that patient. Indeed, we argue that medicine is more than simply applied biology. Knowledge must be gained through diverse technical and subjective experiences acquired over time that affect first-hand interactions with individual patients. This contributes to an understanding of the unique factors that each patient brings to the clinical encounter (9).

It is these domains that allow the physician to appreciate more subtle aspects of the patient's condition, and which may allow nuances in decision-making, and determine the affective or qualitative aspects of a physician's clinical "style" $(10,11)$. Indeed, scientific knowledge alone is sterile, while experiential and contextual knowledge alone is hollow. However, taken together, these forms of knowledge allow the physician to recognize not only that a placebo or nocebo effect has occurred, but to understand why it has occurred, and frame its meaning in the context of a specific clinical encounter. The physician ought to use all of these forms of knowl- edge, balanced by prudent, practical wisdom, to shape the patient-centered decision process that determines what should be done, in contrast to a more mechanistic determination of simply what can be done for a specific patient (7-9).

This raises a second and somewhat more contentious ethical issue. In noting that placebo responses are mechanistically similar to opioid analgesia, the authors qualify the clinical use of placebo as "...not ethically problematic.” This raises questions, at least regarding the underlying rationale and assumptions used to reach this conclusion. Simply because a given treatment engages a set of mechanisms that can elicit a particular outcome does not directly imply that it should be utilized. The use of a sham drug or technique in a patient who is purposely kept uninformed about the nature of the treatment is an explicitly deceptive practice, and therefore a moral error of commission that undermines the practice of medicine. On the surface, the principal focus of this argument is a violation of respect for the autonomy of the patient and the failure to provide information required for patients to consent to treatment. However, the deeper concern is for the dissolution of beneficence-in-trust that forms the basis of the patient-physician relationship $(11,12)$. The patient enters into the medical relationship trusting in the physician's professed knowledge and skill, and is enjoined to the notion that the physician will act to provide a right and good healing. While it may be argued that absolute truthtelling in medicine can be deleterious (13) and that physicians have historically been granted implicit latitude to withhold particular information (14), it is important to recall that veracity is a cornerstone of the trust upon which the medical relationship is built. The intentional use of a sham drug or procedure represents a violation of that trust through the paternalistic manipulation of truth.

A second argument may be that the actual intention is to utilize placebo to produce a positive therapeutic outcome, justifying this position by citing the principle of double effect. However, this is equally problematic in that in order for the principle of double effect to be relevant and applicable, the primary act, that is, the use of sham intervention to produce placebo effects 1) must not be intrinsically wrong, 2) must not simply be a means of producing a good effect, and 3) must have been consented to by the pa- 
tient (15). As matter of fact, as an explicit and intentional deception, the prescriptional use of placebo violates each of these conditions. Moreover, should the nature of the sham intervention be discovered by the patient, the trust necessary for the integrity and potential success of the patient's future medical relationships would be threatened, if not lost altogether.

Yet, Manchikanti, Pampati, and Damron compel us to consider whether placebo effects could be ethically used in clinical practice. We believe this is possible. One way would be for physicians, working within an established clinical relationship, to explain that a particular intervention has not been shown to have inherent properties that are directly therapeutic, but that it has been shown to be effective, and perhaps provide a brief explanation of the possible mechanism(s) involved. Our position is based upon criteria originally proposed by Bok (16) that no blatant lie should be told and that physicians should honestly answer questions about the nature of the treatment if and when asked by patients. As well, we support the work of Jonsen, Siegler, and Winslade (17) in the recommendation to utilize prescriptive placebo only in situations of last resort in which the patient directly requests some form of active care. Obviously, there are additional ethical and medico-legal issues involving the cost of such interventions, although these are beyond the scope of this commentary (a more thorough discussion is provided by Welie (18)).

However, we feel that the most salient point of the work of Manchikanti and colleagues is not a consideration of the use of sham intervention as placebo, but the need to understand the occurrence and nature of placebo and nocebo effects within the context, and perhaps as a consequence of, the clinical encounter itself. This is important in the research setting and in everyday clinical practice.

Brody has described the physician as possessing the capacity to exert a placebo (or nocebo) effect through the strength and integrity of the patient-physician interaction (19). The dynamics involved in producing such effects have been addressed from philosophical (6), ethical $(7,11,12)$, physiologic $(20)$, anthropologic (21) and metaphysical (22) perspectives. Thus, while the interventional pain physician may employ an armamentarium of the most current pharmacological developments and technological advances, these are but one domain of the therapeutic intervention. It is important to recognize that it is often the multi-dimensional understanding of the patient, together with a prudent, empathetic medical rela- tionship, that are critical factors in determining the "valence" of response and the ultimate outcome of treatment.

Author AfFilation:

James Giordano, $\mathrm{PhD}$

Neuroscience section editor, Pain

Physician journal

Research Scholar in Residence

Center for Clinical Bioethics

4000 Reservoir Rd.

Washington, DC 20057

and, Fellow, John P. McGovern MD

Center for Health, Humanities and the Human Spirit

University of Texas Medical Center

Houston, TX

E-mail: gsynapse22@aol.com

\section{Mark V. Boswell, MD, PhD}

Editor-in-Chief, Pain Physician

Associate Professor of Anesthesiology

Chief, Pain Medicine Service

Director, Pain Medicine Fellowship

Department of Anesthesiology

Case Western Reserve School of

Medicine and University Hospitals of

Cleveland

11100 Euclid Ave.

Cleveland, $\mathrm{OH} 44106$

E-mail: mark.boswell@uhhs.com

\section{RefERENCES}

1. Manchikanti L, Pampati VS, Damron K. The role of placebo and nocebo effects of perioperative administration of sedatives and narcotics in interventional pain management. Pain Physician 2005; 8:349-355.

2. Giordano J. The neuroscience of pain and analgesia. In Cole BE, Boswell MV (eds). Weiner's Pain Management: A Guide for Clinicians, seventh edition. Taylor \& Francis, Boca Raton, 2005, pp 15-34.

3. Giordano J. Toward a core philosophy and virtue-based ethics of pain medicine. Pain Practitioner, 2005; 15:59-66.

4. Descartes R. In Anscombe GEM, Geach PT (eds, trans). Descartes: Philosophical Writings. Edinburgh Press, Edinburgh: 1954.

5. Giordano J. Pain research: Can paradigmatic expansion bridge the demands of medicine, scientific philosophy and ethics. Pain Physician 2004; 7:407-410.

6. Svenaeus F. Phenomenology of health and illness. In Toombs SK (ed). Handbook of Phenomenology and Medicine. Kluwer, Amsterdam, 2001, pp 87-108.

7. Pellegrino ED. The healing relationship: Architectonics of clinical medicine. In Shelp EE (ed). The Clinical Encounter: The
Moral Fabric of the Physician-Patient Relationship. Reidel, Boston: 1983.

8. Pellegrino ED. The anatomy of clinical judgments: some notes on right reason and right action. In Engelhardt HT, Spicker SF, Towers B (eds). Clinical Judgment: $A$ Critical Appraisal. Reidel, Dordrecht, 1979, pp 169-194.

9. Aristotle. Nicomachean Ethics, Book VI. Irwin T (trans). Hackett Publishing, Indianapolis, 1999, pp 86-98.

10. Widdershoven-Heerding I. Medicine as a form of practical understanding. Theoretical Med 1987; 8:179-185.

11. Pellegrino ED, Thomasma DC. The Virtues in Medical Practice. Oxford University Press, New York: 1993.

12. Zaner R. Ethics and the Clinical Encounter. Prentice-Hall, Englewood Cliffs: 1988.

13. Gillon R. Telling the truth and medical ethics. Lancet 1985; 291:1556-1557.

14. Bloche MG. Fidelity and deceit at the bedside. I Am Med Assoc 2000; 283:18811884 .

15. Griese ON. The principle of double effect. In Catholic Identity in Health Care: Principles and Practice. The Pope John Center, Braintree, MA, 1987, pp 246-299.
16. Bok S. The ethics of giving placebos. Sc. Am 1974; 231:17-23.

17. Jonsen AR, Siegler M, Winslade WJ. Clinical Ethics, second edition. Macmillan, New York, 1986, pp 69-72.

18. Welie JVM. Placebo treatment. In Encyclopedia of Applied Ethics, Volume 3, 1998, pp 493-502.

19. Brody $\mathrm{H}$. The lie that heals: The ethics of giving placebos. Ann Intern Med 1982; 97: 112-118.

20. Fields HL, Price DD. Toward a neurobiology of placebo analgesia. In Harrington A (ed). The Placebo Effect: An Interdisciplinary Exploration. Harvard University Press, Cambridge, MA, 1997, pp 93-116.

21. Helman CG. Placebos and nocebos: The cultural construction of belief. In Peters D (ed). Understanding the Placebo Effect in Complementary Medicine: Theory Practice and Research. Churchill-Livingstone, Edinburgh, 2001, pp 3-16.

22. Richardson J. Intersubjectivity and the therapeutic relationship. In Peters D (ed). Understanding the Placebo Effect in Complementary Medicine: Theory Practice and Research. Churchill-Livingstone, Edinburgh, 2001, pp 131-146. 